\title{
Formal and Compositional Analysis of Power Systems using Reachable Sets
}

\author{
Matthias Althoff
}

\begin{abstract}
Power system stability analysis becomes more important in the presence of ever increasing variations in operating conditions. Traditionally, the operation of power systems is verified for specific operating conditions. In this work, the stability analysis is performed for a set of operating conditions using reachability analysis, which makes it possible to compute the bounds of all possible system trajectories. Thus, reachability analysis can be used to rigorously check specifications. Contrary to previous work, the presented approach does not require model simplifications when the system is described by semiexplicit, nonlinear, index-1 differential-algebraic equations. The main obstacle in reachability analysis is the scalability towards larger systems, which is addressed by investigating compositional techniques. As a result, transient stability and variable energy production can be analyzed for the IEEE 14-bus and 30-bus benchmark systems, for which the computation times are orders of magnitude faster than the simulation of all cases starting in the corners of the set of possible initial states.
\end{abstract}

Index Terms-Reachability analysis, stability analysis, compositional analysis, power systems, transient stability analysis, uncertain energy production, differential-algebraic equations, formal verification.

\section{INTRODUCTION}

The ongoing trend towards decentralized power generation with a considerable share of renewable energy sources results in a less predictable operation of power systems. New analysis techniques are required to consider all possible future behaviors to ensure a reliable operation of power systems. In this paper, reachability analysis is proposed as a formal technique to verify if specifications are met under uncertain operating conditions. Reachability analysis computes the set of all possible (infinitely many) trajectories of a dynamic model when the uncertainty of initial states, time-varying inputs, and parameters is bounded by sets.

This work focuses on large deviations from the initial operating condition, such that small-signal analysis techniques can no longer be applied [1, Chap. 12]. The dominant technique in power systems for model-based analysis of large disturbances is numerical simulation, which is easy to implement, but can only provide satisfying results when the actual operating condition is known and there are no parametric and input uncertainties. The knowledge of the actual operating condition requires constant simulation of the system for a set of probable contingencies when new SCADA (Supervisory Control And Data Acquisition) measurements are available at a cycle time of around 10-30 minutes [2]. Due to increasingly varying

Matthias Althoff is with the Department of Computer Science, Technische Universität München, 85748 Garching, Germany, email: althoff@tum.de

Manuscript received month day, year; revised month day, year. operating conditions, the measurements at the last cycle might have considerably drifted. In order to rigorously consider those drifts, one has to assume a set of possible initial states covering changes between SCADA updates. However, the number of required simulations grows exponentially with the number of state, input, and parametric variables due to a necessary gridding of the multidimensional set bounding all variables. Besides the exponential complexity, numerical simulation is not a formal technique, i.e., one cannot certify whether the effect of a control action complies with the system specification: 1) it does not show that all states (infinitely many) of an initial set return to the operating point, and 2) it is unclear for how long a simulation has to be run until a particular trajectory can be considered stable. The aforementioned issues are alleviated by faster simulation using parallel-in-space [3] [5] and parallel-in-time algorithms [6] and by Monte Carlo simulation [7]-[9] to address uncertain prediction.

Instead of explicitly simulating the behavior for stability analysis, direct methods compute regions in the state space from which the system state returns to the original operating point [10]-[12]. Those regions are essential to quickly check if control actions are capable of stabilizing the system without requiring time-consuming simulations. Direct methods require Lyapunov functions, which can only be found for simplified system dynamics using network preserving and network reduction methods, where the latter is the dominant technique, see e.g. [10]-[15]. While network preserving methods work with ordinary differential equations (ODEs), network preserving methods use more general models described by differentialalgebraic equations (DAEs) [16]-[19]. A challenge for both is to find the so-called critical value of the Lyapunov function to underapproximate the region of attraction. Especially for systems with several generators, the critical value is rather conservative, resulting in an underapproximation of the region of attraction [10], [11]. Another disadvantages of direct methods is that one cannot check if phase, voltage, and frequency constraints are met since direct methods only analyze if a steady state of a disturbed system is eventually reached.

Reachability analysis is a complementary analysis technique besides simulation techniques and direct methods. Differing from simulation techniques, reachability analysis can prove whether system specifications are fulfilled in the presence of uncertainties, such as uncertain initial states and uncertain inputs/disturbances. Direct methods are a formal technique since they can guarantee stability when the initial state starts in the computed region of attraction. However, other than reachability analysis, direct methods cannot formally verify whether constraints are met (e.g. whether frequencies and voltages remain within permitted ranges). Since formal analysis of 
the nonlinear dynamics of power systems is undecidable [20], one cannot compute exact reachable sets for this system class, requiring instead the computation of an overapproximation of the reachable set, which includes all behaviors of the modeled system. However, when the overapproximation is too large, one might not be able to verify the system although all specifications are met in reality. General literature reviews on reachability analysis of dynamic systems can be found in [21] [24]. Most previous work on reachability analysis in power systems has been limited to small problems due to the initial computational costs of first attempts. In [25], [26], reachability analysis is performed for a single-machine-infinite-bus system modeled by ordinary differential equations with only 2 state variables. A slightly larger double-machine-infinite-bus system with 2 buses described by ODEs resulting in 5 state variables is considered in [27], where simulations are performed to approximate the reachable set resulting in a non-formal approach, i.e. one cannot prove that the controller meets the specifications under all eventualities. A 3-bus system is considered in [28] to investigate effects on wind variability. More recent work of the same authors considers the effects of wind variability for the 39-bus New England system model [29] and similar studies on the effect of uncertain energy production on frequency deviation are studied in [30] for a reduced-order model of the U.S. power system. The computations in [28]-[30] are simplified by linearizing the system dynamics so that the results are not overapproximative anymore and thus do not qualify for formal analysis.

To the best knowledge of the author, none of the previous methods for reachability analysis of power systems considers the original DAE system arising in power system modeling, instead relying on simplification of the system dynamics to ordinary differential equations. In the previous work of the author [31], a new method is presented which can compute the reachable set of the original DAE systems. The approach proposed in [31] has a complexity of $\mathcal{O}\left(n^{5}\right)$, where $n$ is the number of state variables. Although the complexity is polynomial, the analysis of large systems results in enormous computational costs. In this work, the computational costs are drastically reduced by investigating compositional techniques, making it possible to compute reachable sets of the IEEE 30bus benchmark system in less time compared to the IEEE 14-bus version when no compositional techniques are applied. Another extension compared to [31] and other previous works is the investigation of the effect of uncertain energy production-not only on frequency, but also on bus voltage and phase under consideration of nonlinear effects.

\section{PROBlem Formulation}

We consider power systems that can be modeled as a set of semi-explicit, nonlinear, index-1 DAEs, which applies to almost all power systems (see e.g. [32]). For brevity of the presentation it is assumed that the parameters of the power system are known and constant over time, resulting in a set of timeinvariant DAEs. Extensions required for uncertain parameters are presented in [33]. The vectors of differential variables, algebraic variables, and inputs are respectively denoted by $x \in \mathbb{R}^{n_{d}}, y \in \mathbb{R}^{n_{a}}$, and $u \in \mathbb{R}^{m}$, where $n_{d}, n_{a}$, and $m$ are the corresponding numbers of variables. For a set of consistent initial states $\mathcal{R}(0)$ and a set of possible inputs/disturbances $\mathcal{U}$, the system equations are

$$
\begin{aligned}
\dot{x} & =f(x(t), y(t), u(t)), \\
0 & =g(x(t), y(t), u(t)),
\end{aligned}
$$

where $\left[x^{T}(0), y^{T}(0)\right]^{T} \in \mathcal{R}(0), u(t) \in \mathcal{U}$. The initial state is consistent when $g(x(0), y(0), u(0))=0$ and it is assumed that (1) has a unique solution $\gamma(t, x(0), y(0), u(\cdot))$ for all consistent initial states $x(0), y(0)$ and all piecewise continuous input trajectories $u(\cdot)$, where $u(t)$ refers to an input at a specific point in time $t$. No other assumption besides unique solutions are required. The goal of this work is to compute the reachable set $\mathcal{R}^{e}\left(\left[0, t_{f}\right]\right)$ of (1) for a time interval $\left[0, t_{f}\right]$ :

$$
\begin{aligned}
\mathcal{R}^{e}\left(\left[0, t_{f}\right]\right)=\{ & \gamma(t, x(0), y(0), u(\cdot)) \mid\left[x^{T}(0), y^{T}(0)\right]^{T} \in \mathcal{R}(0), \\
& \left.u(t) \in \mathcal{U}, t \in\left[0, t_{f}\right]\right\} .
\end{aligned}
$$

The superscript $e$ on $\mathcal{R}^{e}\left(\left[0, t_{f}\right]\right)$ denotes the exact reachable set, which cannot be computed for nonlinear DAE systems as mentioned in the introduction [20]. For this reason, algorithms are presented which compute as tight as possible overapproximations $\mathcal{R}\left(\left[0, t_{f}\right]\right) \supseteq \mathcal{R}^{e}\left(\left[0, t_{f}\right]\right)$. For simplification, the expression reachable set is used even when overapproximative reachable sets are computed. For later derivations, the projection of the reachable set onto the coordinates of differential variables is denoted by $\mathcal{R}^{d}\left(\left[0, t_{f}\right]\right)$ and onto the algebraic variables by $\mathcal{R}^{a}\left(\left[0, t_{f}\right]\right)$. Since reachable sets contain the union of all possible simulations, the same types of analysis performed with simulations can be performed with reachable sets. Note that reachability can in principle also be applied to chaotic systems [34]. In this work, the focus is on transient stability analysis and the effects of uncertain energy production.

\section{Preliminaries}

The presented approach is based on known techniques for computing reachable sets of linear differential inclusions, which are recapitulated in this section. Reachable set computations are typically performed iteratively for short time intervals

$$
\tau_{k}:=\left[t_{k}, t_{k+1}\right] .
$$

In this work, constant-size time intervals $t_{k}:=k r$ are used to focus on the main innovations, where $k \in \mathbb{N}$ is the time step and $r \in \mathbb{R}^{+}$is referred to as the time increment. An extension to variable time increments is described in [35].

The iterative computation of reachable sets for linear systems requires set-based addition or Minkowski addition $(\mathcal{X} \oplus \mathcal{Y}:=\{x+y \mid x \in \mathcal{X}, y \in \mathcal{Y}\})$ and set-based multiplication $(\mathcal{X} \otimes \mathcal{Y}:=\{x y \mid x \in \mathcal{X}, y \in \mathcal{Y}\})$. Note that the symbol for set-based multiplication is often omitted for simplicity of notation, and that one or both operands can be singletons. A brief description of the main steps for obtaining reachable sets for a single time interval is provided below.

In this section, the reachability analysis of linear differential inclusion $\dot{\tilde{x}} \in \tilde{A} \tilde{x}(t) \oplus \tilde{\mathcal{U}}$ is recapitulated, where $\tilde{x} \in \mathbb{R}^{n_{d}}$, $\tilde{A} \in \mathbb{R}^{n_{d} \times n_{d}}$, and $\tilde{\mathcal{U}} \subset \mathbb{R}^{n_{d}}$ is a set of uncertain inputs. A 
tilde is used for the variables of the linear differential inclusion to distinguish the variables from the ones of the original nonlinear DAEs. For further computations, some variables and sets are introduced: $u_{c}$ is the center of $\tilde{\mathcal{U}}, \tilde{\mathcal{U}}_{\Delta}:=\tilde{\mathcal{U}} \oplus\left(-u_{c}\right)$ is the deviation of $\tilde{\mathcal{U}}$ from the center $u_{c}$, the reachable set of the affine dynamics $\dot{\tilde{x}}=\tilde{A} \tilde{x}(t)+u_{c}$ is $\mathcal{R}_{a}^{d}(t)$, the reachable set of the particular solution due to the uncertain input $\tilde{\mathcal{U}}_{\Delta}$ is $\mathcal{R}_{p}^{d}\left(\tilde{\mathcal{U}}_{\Delta}, t\right)$, and the partial reachable set correcting the initial assumption that trajectories are straight lines between $t_{k}$ and $t_{k+1}$ is $\mathcal{R}_{\epsilon}^{d}$. According to [23], the reachable set for a time interval $\tau_{k}$ is computed as shown in Fig. 1:

1) Starting from $\mathcal{R}^{d}\left(t_{k}\right)$, compute the set of all solutions $\mathcal{R}_{a}^{d}\left(t_{k+1}\right)$ for the affine dynamics $\dot{\tilde{x}}=\tilde{A} \tilde{x}(t)+u_{c}$ at time $t_{k+1}$.

2) Obtain the convex hull of $\mathcal{R}^{d}\left(t_{k}\right)$ and $\mathcal{R}_{a}^{d}\left(t_{k+1}\right)$ to approximate the reachable set for the time interval $\tau_{k}$.

3) Compute $\mathcal{R}^{d}\left(\tau_{k}\right)$ by considering uncertain inputs by adding $\mathcal{R}_{p}^{d}\left(\tilde{\mathcal{U}}_{\Delta}, r\right)$ and accounting for the curvature of trajectories by adding $\mathcal{R}_{\epsilon}^{d}$.

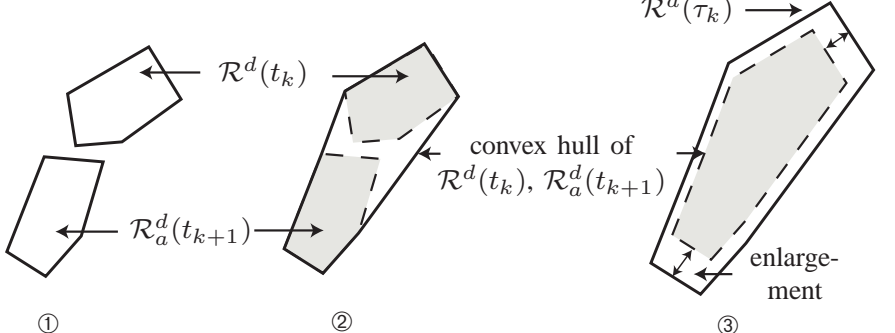

Fig. 1. Steps for computing the reachable set for a linear system.

Using $r=t_{k+1}-t_{k}$, the solution of $\mathcal{R}_{a}^{d}\left(t_{k+1}\right)$ is

$$
\mathcal{R}_{a}^{d}\left(t_{k+1}\right)=e^{\tilde{A} r} \mathcal{R}^{d}\left(t_{k}\right)+\underbrace{\int_{0}^{r} e^{\tilde{A}(r-t)} d t u_{c}}_{=: x_{p}(r)},
$$

where $x_{p}(r)$ is bounded by integrating the finite Taylor series $e^{\tilde{A} r}=\sum_{i=0}^{\eta}(\tilde{A} r)^{i} /(i !)$ up to order $\eta$ to which the remainder $\mathcal{E}_{p}(r)$ is added:

$$
x_{p}(r) \in \underbrace{\left(\sum_{i=0}^{\eta} \frac{\tilde{A}^{i} r^{i+1}}{(i+1) !} \oplus \mathcal{E}_{p}(r)\right)}_{=: \Gamma(r)} u_{c} .
$$

The remainder can be overapproximated by an interval matrix $\mathcal{E}_{p}(r):=[-W(r) r, W(r) r]$, i.e., by a matrix with lower and upper bounds on each element, where $W(r)=e^{|\tilde{A}| r}-$ $\sum_{i=0}^{\eta} \frac{\mid \tilde{A} i^{i} r^{i}}{i !}$. For later derivations, $\tilde{W}(r):=W(r) r$ is also introduced. The required enlargement of the convex hull (see $3^{\text {rd }}$ step in Fig. 1) is achieved by adding $\mathcal{R}_{\epsilon}^{d}$ to account for the curvature of trajectories from $\mathcal{R}^{d}\left(t_{k}\right)$ to $\mathcal{R}_{a}^{d}\left(t_{k+1}\right)$ (see [23]) and by adding the reachable set $\mathcal{R}_{p}^{d}\left(\tilde{\mathcal{U}}_{\Delta}, r\right)$ due to the uncertain and convex input set $\tilde{\mathcal{U}}_{\Delta}$ (see [36]):

$$
\begin{aligned}
\mathcal{R}_{\epsilon}^{d} & :=\left(\mathcal{F} \otimes \mathcal{R}^{d}\left(t_{k}\right)\right) \oplus\left(\tilde{\mathcal{F}} \otimes u_{c}\right) \\
\mathcal{R}_{p}^{d}\left(\tilde{\mathcal{U}}_{\Delta}, r\right) & :=\bigoplus_{i=0}^{\eta}\left(\frac{\tilde{A}^{i} r^{i+1}}{(i+1) !} \tilde{\mathcal{U}}_{\Delta}\right) \oplus\left([-\tilde{W}(r), \tilde{W}(r)] \otimes\left|\tilde{\mathcal{U}}_{\Delta}\right|\right),
\end{aligned}
$$

with

$$
\begin{aligned}
& \mathcal{F}:=\left(\bigoplus_{i=2}^{\eta}\left[\left(i^{\frac{-i}{i-1}}-i^{\frac{-1}{i-1}}\right) r^{i}, 0\right] \frac{\tilde{A}^{i}}{i !}\right) \oplus[-W(r), W(r)] \\
& \tilde{\mathcal{F}}:=\left(\bigoplus_{i=2}^{\eta+1}\left[\left(i^{\frac{-i}{i-1}}-i^{\frac{-1}{i-1}}\right) r^{i}, 0\right] \frac{\tilde{A}^{i-1}}{i !}\right) \oplus[-\tilde{W}(r), \tilde{W}(r)] .
\end{aligned}
$$

The absolute value $\left|\tilde{\mathcal{U}}_{\Delta}\right|_{i}:=\sup \left\{\left|u_{i}\right| \mid u \in \tilde{\mathcal{U}}_{\Delta}\right\}$ is defined elementwise. The reachable sets for the next point in time and time interval are obtained by combining all previous results (see [36]):

$$
\begin{aligned}
\mathcal{R}^{d}\left(t_{k+1}\right):= & e^{A r} \mathcal{R}^{d}\left(t_{k}\right) \oplus \Gamma(r) u_{c} \oplus \mathcal{R}_{p}^{d}\left(\tilde{\mathcal{U}}_{\Delta}, r\right), \\
\mathcal{R}^{d}\left(\tau_{k}\right):= & \operatorname{CH}\left(\mathcal{R}^{d}\left(t_{k}\right), e^{A r} \mathcal{R}^{d}\left(t_{k}\right) \oplus \Gamma(r) u_{c}\right) \\
& \oplus \mathcal{R}_{\epsilon}^{d} \oplus \mathcal{R}_{p}^{d}\left(\tilde{\mathcal{U}}_{\Delta}, r\right),
\end{aligned}
$$

where $\mathrm{CH}()$ returns the convex hull. Throughout this work, zonotopes are used to represent the reachable sets. However, the proposed algorithms and computations apply to all kinds of set representations. Zonotopes are used since they can efficiently represent reachable sets in high-dimensional spaces while operations required for reachability analysis can efficiently be applied to them. Details on the definition of zonotopes and operations on them are described in Sec. VI.

\section{Reachability Analysis of Nonlinear DAE SYSTEMS}

Other than for linear systems, no closed-form solution exists for general nonlinear DAEs. In order to exploit the efficient methods of the previous section based on the closedform solution of linear systems, an abstraction of the original nonlinear DAEs to linear differential inclusions is performed for each consecutive time interval $\tau_{k}$. The linear differential inclusions are computed such that the resulting abstraction is strictly overapproximative, i.e. it contains all behaviors of the original dynamics. By re-computing the abstraction for each time interval, the overapproximation remains small and accurate results are obtained while traversing the nonlinear state space far away from the original operating point.

\section{A. Abstraction to Linear Differential Inclusions}

The abstraction of the nonlinear DAEs to linear differential inclusions is based on linearizing the system dynamics and adding the linearization errors as uncertain input. For a concise notation, the vector $z:=\left[x^{T}, y^{T}, u^{T}\right]^{T}$, the linearization point $z^{*}:=\left[x^{* T}, y^{* T}, u^{* T}\right]^{T}$, and $\mathcal{R}^{z}:=\mathcal{R}\left(\tau_{k}\right) \times \mathcal{U}$ are introduced. To efficiently obtain a suitable linearization point, the volumetric centers $c^{d}, c^{a}, c^{u}$ of the sets $\mathcal{R}^{d}\left(t_{k}\right), \mathcal{R}^{a}\left(t_{k}\right)$, and $\mathcal{U}$, are introduced. In a previous work it is shown that the center of the reachable set of the current time interval is the best linearization point when the linearization error is computed via an evaluation of the Lagrange remainder using bounds on absolute values [33, Prop. 2]. Although a refined technique is used for the linearization error computation in this work, the center of the reachable set remains the best choice for the linearization error. To circumvent the problem 
that the reachable set is required for an optimal linearization point, which in turn is required for the computation of the reachable set, the center of the differential variables is approximated by a one-step Euler integration. This yields $x^{*}=c^{d}+0.5 r \cdot f\left(c^{d}, c^{a}, c^{u}\right)$, where the step size is $0.5 r$ because the center of the reachable set $\mathcal{R}^{d}\left(\tau_{k}\right)$ for the time interval $\tau_{k}$ is expected to be reached after half the interval duration of $t_{k+1}-t_{k}=r$ from the center $c^{d}$ of $\mathcal{R}^{d}\left(t_{k}\right)$. Besides the linearization point of the dynamic variables, the linearization point of the inputs is chosen as $u^{*}=c^{u}$ and the linearization point of the algebraic part is computed such that it is consistent with the constraint $0=g\left(x^{*}, y^{*}, u^{*}\right)$ using the Newton-Raphson method. The linearization of the original dynamics in (1) is performed using a first-order Taylor expansion with Lagrangian remainder:

$$
\begin{aligned}
& \dot{x}_{i}=f_{i}(z(t)) \in f_{i}\left(z^{*}\right)+\left.\frac{\partial f_{i}(z)}{\partial z}\right|_{z=z^{*}}\left(z(t)-z^{*}\right) \\
& \oplus \underbrace{\left\{\left.\frac{1}{2}\left(z(t)-z^{*}\right)^{T} \frac{\partial^{2} f_{i}(z)}{\partial z^{2}}\right|_{z=\xi}\left(z(t)-z^{*}\right) \mid \xi, z(t) \in \mathcal{R}^{z}\right\}}_{=: \mathcal{L}_{i}^{d}}, \\
& 0=g_{j}(z(t)) \in g_{j}\left(z^{*}\right)+\left.\frac{\partial g_{j}(z)}{\partial z}\right|_{z=z^{*}}\left(z(t)-z^{*}\right) \\
& \oplus \underbrace{\left\{\left.\frac{1}{2}\left(z(t)-z^{*}\right)^{T} \frac{\partial^{2} g_{j}(z)}{\partial z^{2}}\right|_{z=\xi}\left(z(t)-z^{*}\right) \mid \xi, z(t) \in \mathcal{R}^{z}\right\}}_{=: \mathcal{L}_{j}^{a}},
\end{aligned}
$$

where $\mathcal{L}_{i}^{d}$ denotes the projection of $\mathcal{L}^{d}$ onto the $i^{\text {th }}$ coordinate. The Lagrangian remainders $\mathcal{L}^{d}$ and $\mathcal{L}^{a}$ enclose all higherorder terms if $z^{*}, \xi, z(t) \in \mathcal{R}^{z}$ [37, p. 87]. For subsequent derivations, it is required to separate the effects from differential variables, algebraic variables, and inputs. Therefor, the following sub-matrices of the Jacobians are introduced:

$$
\left.\frac{\partial f(z)}{\partial z}\right|_{z=z^{*}}=[A, C, B],\left.\quad \frac{\partial g(z)}{\partial z}\right|_{z=z^{*}}=[D, F, E],
$$

where $A \in \mathbb{R}^{n_{d} \times n_{d}}, B \in \mathbb{R}^{n_{d} \times m}, C \in \mathbb{R}^{n_{d} \times n_{a}}, D \in$ $\mathbb{R}^{n_{a} \times n_{d}}, E \in \mathbb{R}^{n_{a} \times m}, F \in \mathbb{R}^{n_{a} \times n_{a}}$, and $n_{d}, n_{a}, m$ are the number of differential, algebraic, and input variables, respectively. Inserting the abbreviation $z=\left[x^{T}, y^{T}, u^{T}\right]^{T}$ and the matrices $A-F$ into (3), and introducing $H^{d,(i)}(\xi):=$ $\left.\left.\frac{\partial^{2} f_{i}(z)}{\partial z^{2}}\right)\left.\right|_{z=\xi}, H^{a,(j)}(\xi):=\frac{\partial^{2} g_{j}(z)}{\partial z^{2}}\right)\left.\right|_{z=\xi}, \mathcal{R}_{\Delta}^{z}:=\mathcal{R}^{z} \oplus\left(-z^{*}\right)$, $\nu(t):=z(t)-z^{*}$, yields

$$
\begin{gathered}
\dot{x} \in f\left(z^{*}\right)+A(\underbrace{x(t)-x^{*}}_{=: \Delta x(t)})+B(\underbrace{u(t)-u^{*}}_{=: \Delta u(t)})+C(\underbrace{y(t)-y^{*}}_{=: \Delta y(t)}) \\
\oplus\left\{\frac{1}{2} \sigma \mid \sigma_{i}=\nu^{T} H^{d,(i)}(\xi) \nu, \xi \in \mathcal{R}^{z}, \nu \in \mathcal{R}_{\Delta}^{z}\right\}, \\
0 \in g\left(z^{*}\right)+D(\underbrace{x(t)-x^{*}}_{=: \Delta x(t)})+E(\underbrace{u(t)-u^{*}}_{=: \Delta u(t)})+F(\underbrace{y(t)-y^{*}}_{=: \Delta y(t)}) \\
\oplus\left\{\frac{1}{2} \phi \mid \phi_{j}=\nu^{T} H^{a,(j)}(\xi) \nu, \xi \in \mathcal{R}^{z}, \nu \in \mathcal{R}_{\Delta}^{z}\right\} .
\end{gathered}
$$

Note that $F$ is invertible because of the index-1 property, so that one can reformulate (6) to

$$
\begin{gathered}
\Delta y(t) \in-F^{-1}\left(g\left(z^{*}\right)+D \Delta x(t)+E \Delta u(t)\right) \\
\oplus\left\{-\frac{1}{2} F^{-1} \phi \mid \phi_{j}=\nu^{T} H^{a,(j)}(\xi) \nu, \xi \in \mathcal{R}^{z}, \nu \in \mathcal{R}_{\Delta}^{z}\right\} .
\end{gathered}
$$

Inserting (7) into (5) results in a differential inclusion

$$
\begin{aligned}
\dot{x} \in & f\left(z^{*}\right)+A \Delta x(t)+B \Delta u(t) \\
& -C F^{-1}\left(g\left(z^{*}\right)+D \Delta x(t)+E \Delta u(t)\right) \oplus \mathcal{L} \\
= & (w+\tilde{A} \Delta x(t)+\tilde{B} \Delta u(t)) \oplus \mathcal{L},
\end{aligned}
$$

where

$$
\begin{gathered}
w:=f\left(z^{*}\right)-C F^{-1} g\left(z^{*}\right), \\
\tilde{A}:=A-C F^{-1} D, \quad \tilde{B}:=B-C F^{-1} E,
\end{gathered}
$$

and

$$
\begin{aligned}
\mathcal{L}= & \left\{\frac{1}{2}\left(\sigma-C F^{-1} \phi\right) \mid \sigma_{i}=\nu^{T} H^{d,(i)}(\xi) \nu,\right. \\
& \left.\phi_{j}=\nu^{T} H^{a,(j)}(\xi) \nu, \xi \in \mathcal{R}^{z}, \nu \in \mathcal{R}_{\Delta}^{z}\right\} .
\end{aligned}
$$

One can further simplify (8) by combining the singleton $w$ and the sets $\tilde{B}\left(\mathcal{U} \oplus\left(-u^{*}\right)\right)$ and $\mathcal{L}$ to a new set $\tilde{\mathcal{U}}$ :

$$
\begin{gathered}
\dot{\tilde{x}} \in \tilde{A} \tilde{x}(t) \oplus \tilde{\mathcal{U}}, \\
\tilde{x}(t):=\Delta x(t), \quad \tilde{\mathcal{U}}:=w \oplus \tilde{B}\left(\mathcal{U} \oplus\left(-u^{*}\right)\right) \oplus \mathcal{L} .
\end{gathered}
$$

The obtained differential inclusion can be solved as described in Sec. III. Still remaining is the determination of linearization errors.

\section{B. Computation of the Linearization Error}

The problem with evaluating (11) is that the set of linearization errors $\mathcal{L}$ is not known in advance, consequently $\tilde{\mathcal{U}}$ is unknown, as well. As an initial guess the most recently computed linearization error $\tilde{\mathcal{L}}$ is enlarged by a user-defined factor $\lambda_{L} \in \mathbb{R}^{+}$around the volumetric center $\hat{c}_{L}$ of $\tilde{\mathcal{L}}$ :

$$
\overline{\mathcal{L}}=\hat{c}_{L} \oplus \lambda_{L}\left(\tilde{\mathcal{L}} \oplus\left(-\hat{c}_{L}\right)\right) .
$$

In the event that the enclosure assumption $(\overline{\mathcal{L}} \supseteq \mathcal{L})$ is not correct after computing the reachable set and the associated set of linearization errors, $\overline{\mathcal{L}}$ has to be further enlarged. In order to bound the set of linearization errors, one additionally checks if $\overline{\mathcal{L}} \subseteq \mathcal{L}_{\text {max }}$, where $\mathcal{L}_{\text {max }}$ is set by the user. If the above inclusion is not fulfilled, the reachable set has to be split in order to reduce the linearization error or the time increment $r$ has to be reduced.

The set of linearization errors $\mathcal{L}$ is computed based on the reachable set $\mathcal{R}^{d}\left(\tau_{k}\right)$ of the linear differential inclusion (11) after replacing the input set $\tilde{\mathcal{U}}$ by $\overline{\mathcal{U}}=w \oplus \tilde{B}\left(\mathcal{U} \oplus\left(-u^{*}\right)\right) \oplus \overline{\mathcal{L}}$, which considers the linearization error assumption $\overline{\mathcal{L}}$ instead of $\mathcal{L}$. After applying the procedures in Sec. III on the linear differential inclusion (11), one obtains the reachable set of the differential variables $\mathcal{R}^{d}\left(\tau_{k}\right)$. For computing the set of linearization errors, the reachable set of the differential and algebraic variables is required, which can be solely reconstructed by the reachable set of the differential variables. This 
is achieved by firstly starting with $x(t)=x^{*}+\Delta x(t)$ and $y(t)=y^{*}+\Delta y(t)$, secondly replacing $\Delta y(t)$ with (7), and thirdly substituting specific values by sets:

$$
\begin{aligned}
\mathcal{R}\left(\tau_{k}\right)= & {\left[\begin{array}{c}
x^{*} \\
y^{*}-F^{-1} g\left(z^{*}\right)
\end{array}\right] \oplus\left[\begin{array}{c}
I \\
-F^{-1} D
\end{array}\right]\left(\mathcal{R}^{d}\left(\tau_{k}\right)-x^{*}\right) } \\
& \oplus\left[\begin{array}{c}
\mathbf{0} \\
-F^{-1} E
\end{array}\right]\left(\overline{\mathcal{U}}-u^{*}\right) \oplus\left[\begin{array}{c}
\mathbf{0} \\
-F^{-1}
\end{array}\right] \overline{\mathcal{L}}^{a} .
\end{aligned}
$$

The reachable set $\overline{\mathcal{R}}\left(\tau_{k}\right)$ is used to compute the set of linearization errors $\mathcal{L}$ as presented in [31]. The computation of the linearization error is the bottleneck in terms of computational costs of the presented approach. When using zonotopes as a set representation, all operations required for the reachability analysis excluding the linearization error computation have complexity $\mathcal{O}\left(n^{3}\right)$, where $n$ is the number of dynamic and algebraic variables. The linearization error computation, however, has complexity $\mathcal{O}\left(n^{5}\right)$. For this reason, compositional techniques are investigated to scale the approach to larger power systems as presented in the next section. The overall algorithm of all previously mentioned steps is presented in Alg. 1.

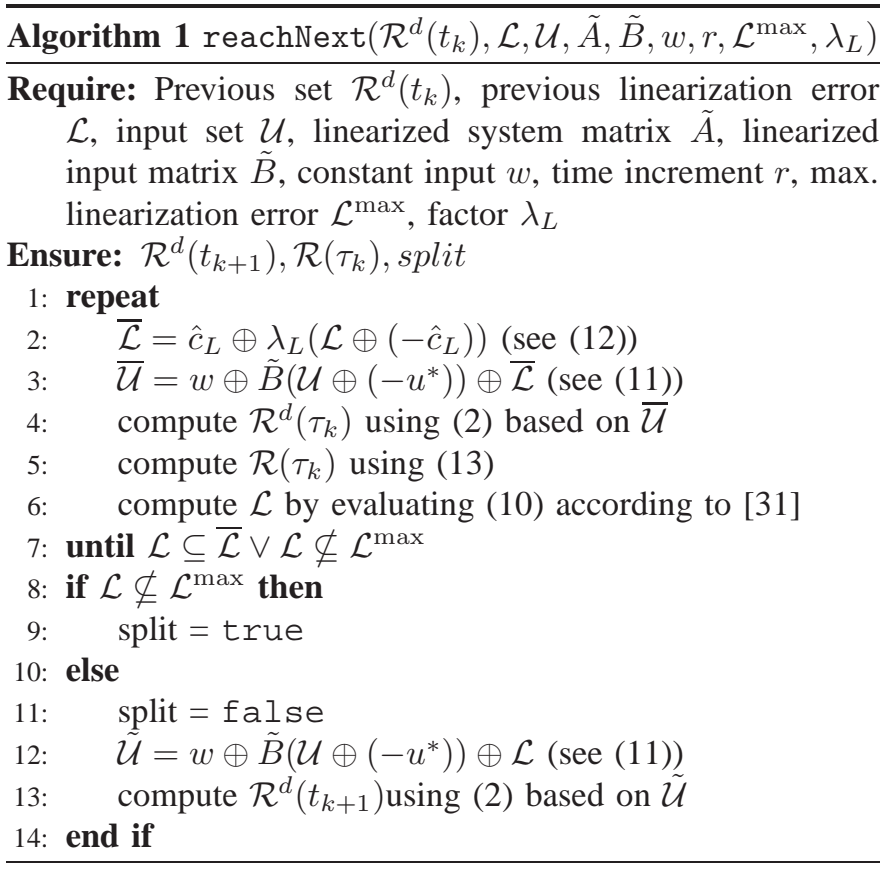

\section{Compositional Reachable Set Computation}

In order to improve the scalability of the proposed reachability analysis, two compositional techniques are investigated. One of them is to split the power grid into subsystems for which the reachable set is computed separately. The other technique compositionally computes the set of linearization errors, while abstracting the dynamics to linear differential inclusions using the full model.

Before applying compositional analysis techniques, one has to partition the power system into subsystems. Partitioning of power systems is a well-known problem for different aspects of power systems. Examples include coherency-based decomposition [38], [39] and graph-based decomposition [40]. Since this work focuses on the compositional computation of reachable sets for a given partition, we assume that a reasonable partition is already provided, where transmission lines are the interfaces. Since the transmission lines are the subsystem interfaces, the bus phase angles and the bus voltage are internal variables when the bus is within the subsystem, and a system input otherwise. The assignment of the variables to the dynamic and algebraic state vectors $x, y$ as well as to the input vector $u$ are described in Sec. VII-A.

\section{A. Compositional Reachable Set Computation}

This subsection describes the first option investigated for compositional analysis by splitting the power system into subsystems. The reachable set of each subsystem is computed as presented in Alg. 1. The input sets representing inputs to the complete system are known, however, the input sets originating from the interfaces of the subsystem are unknown. They depend on the reachable set of neighboring subsystems, which in turn depend on the reachable sets of other subsystems. The basic idea for breaking this mutual dependence apart is similar to the computation of the linearization error. First, the interface inputs of the $i^{\text {th }}$ subsystems are enlarged by a factor $\lambda_{U}$ (analogously to (12))

$$
\hat{\mathcal{U}}^{(i)}=\hat{c}_{U}^{(i)} \oplus\left(\left(\lambda_{U}-1\right) \Lambda^{(i)}+I\right)\left(\mathcal{U}^{(i)} \oplus\left(-\hat{c}_{U}^{(i)}\right)\right),
$$

where $\Lambda$ is a diagonal matrix that contains ones for indices corresponding to interface inputs and zeros otherwise. Based on the system input $\hat{\mathcal{U}}^{(i)}$, the reachable set of the corresponding subsystem is computed as presented in Alg. 1. For aggregating the reachable sets of the complete system by partial reachable sets $\mathcal{R}^{(i)}\left(\tau_{k}\right)$ of the $i^{\text {th }}$ subsystem, matrices $\Phi^{(i)}$ are introduced, which map the local states of the $i^{\text {th }}$ subsystem to the states of the full system. The matrices $\Phi^{(i)}$ contain ones when states are correlated, and zeros otherwise, so that the complete reachable set is obtained using the Cartesian product:

$\mathcal{R}\left(\tau_{k}\right)=\Phi^{(1)} \mathcal{R}^{(1)}\left(\tau_{k}\right) \times \Phi^{(2)} \mathcal{R}^{(2)}\left(\tau_{k}\right) \times \ldots \times \Phi^{\left(n_{s}\right)} \mathcal{R}^{\left(n_{s}\right)}\left(\tau_{k}\right)$,

where $n_{s}$ is the number of subsystems and the computation of linear maps and Cartesian products of zonotopes is performed as presented in Sec. VI. In order to check if the assumption on the set of interface inputs is correct for all subsystems, further matrices $\Upsilon^{(i)}$ are introduced, which map the states of the complete system to the interface inputs of the $i^{\text {th }}$ subsystem. Again, the matrix contains ones for corresponding states and interface inputs and zeros otherwise. If

$$
\forall i: \Upsilon^{(i)} \mathcal{R}\left(\tau_{k}\right) \subseteq \Lambda^{(i)} \hat{\mathcal{U}}^{(i)}
$$

the assumption is overapproximative and thus valid. Otherwise, one has to re-apply the enlargement in (14) for the subsystems that violate the assumption. This procedure is summarized in Alg. 2 under the assumption that no split is required. 


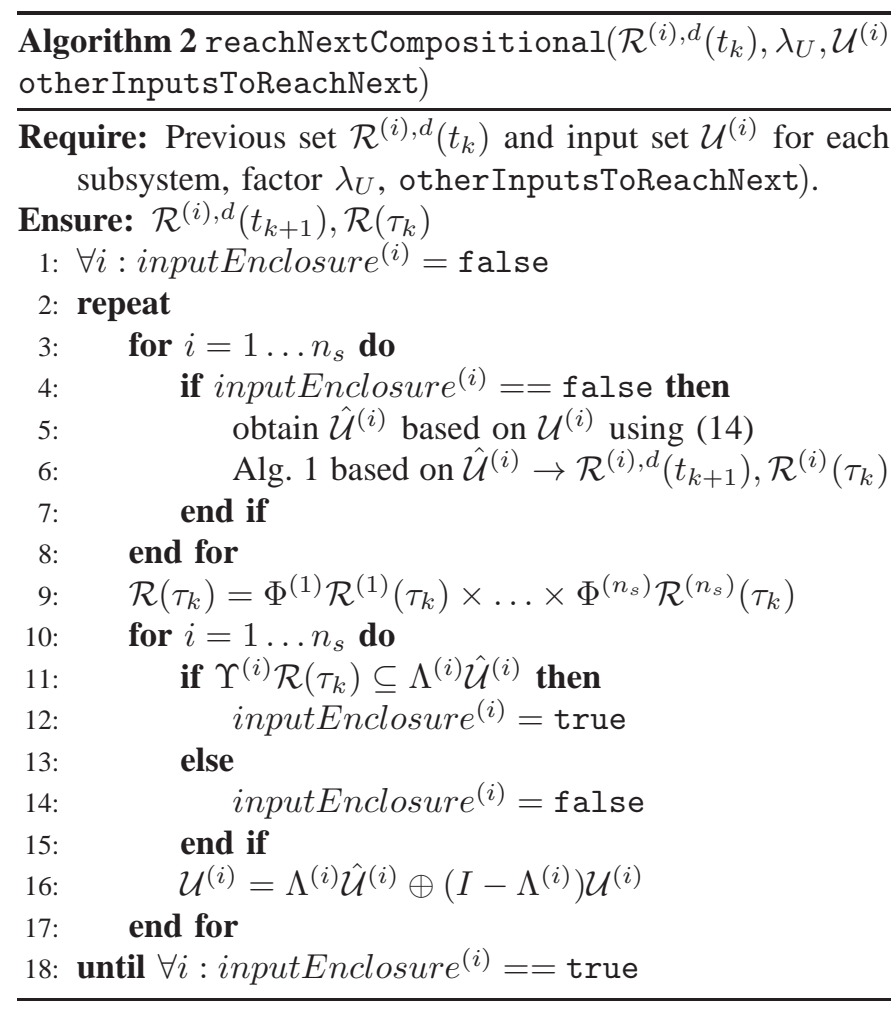

\section{B. Compositional Linearization Error Computation}

In some power systems, generators might be strongly correlated, resulting in unsatisfactory overapproximations of the compositional algorithm in Alg. 2. Since most of the computation time is spent on evaluating the linearization error, one could only compute the linearization error compositionally, while maintaining all the correlations for the reachable set computation in Alg. 1.

Using a decomposition of the full system into subsystems as in the previous subsection, the Lagrangian remainder in (10) is evaluated compositionally. For this purpose, the set of input values from subsystem interfaces has to be considered resulting in the set of inputs for each subsystem as proposed in (14). The partial Lagrange remainders of the $i^{\text {th }}$ subsystem denoted by $\mathcal{L}^{(i)}$ are combined to the complete Lagrange remainder as for the reachable set in (15):

$\mathcal{L}\left(\tau_{k}\right)=\Phi^{(1)} \mathcal{L}^{(1)}\left(\tau_{k}\right) \times \Phi^{(2)} \mathcal{L}^{(2)}\left(\tau_{k}\right) \times \ldots \times \Phi^{\left(n_{s}\right)} \mathcal{L}^{\left(n_{s}\right)}\left(\tau_{k}\right)$.

As previously mentioned, the Lagrange remainder has complexity $\mathcal{O}\left(n^{5}\right)$, where $n$ is the number of state variables, whereas all other operations have complexity $\mathcal{O}\left(n^{3}\right)$ when using zonotopes as the set representation. Thus, the compositional computation of the linearization error has similar computational savings than the completely compositional computation as presented in the previous subsection.

\section{Set Representation by Zonotopes}

So far, all set-based computations have been introduced independently of the set representation so that all kinds of set representations can be used in principle. Typical set representations are: polytopes [41], zonotopes [42], ellipsoids [43], support functions [44], and oriented hyperrectangles [45]. As shown in Sec. III, the set operations required for reachability analysis of linear systems are matrix and interval matrix multiplication, Minkowski addition, absolute value computation, and convex hull. All of these can be efficiently computed using zonotopes, which makes zonotopes very attractive for reachability computations of linear systems [42], [46].

Definition VI.1 (Zonotope) Given a center $c \in \mathbb{R}^{n}$ and socalled generators $g^{(i)} \in \mathbb{R}^{n}$, a zonotope is defined as

$$
\mathcal{Z}:=\left\{x \in \mathbb{R}^{n} \mid x=c+\sum_{i=1}^{p} \beta_{i} g^{(i)}, \beta_{i} \in[-1,1]\right\}
$$

We write in short $\mathcal{Z}=\left(c, g^{(1)}, \ldots, g^{(p)}\right)$ and define the order of a zonotope as $\rho:=\frac{p}{n}$, where $p$ is the number of generators. A zonotope can be seen as the Minkowski addition of line segments $[-1,1] g^{(i)}$, which provides an intuition of how a zonotope is constructed as presented in Fig. 2.

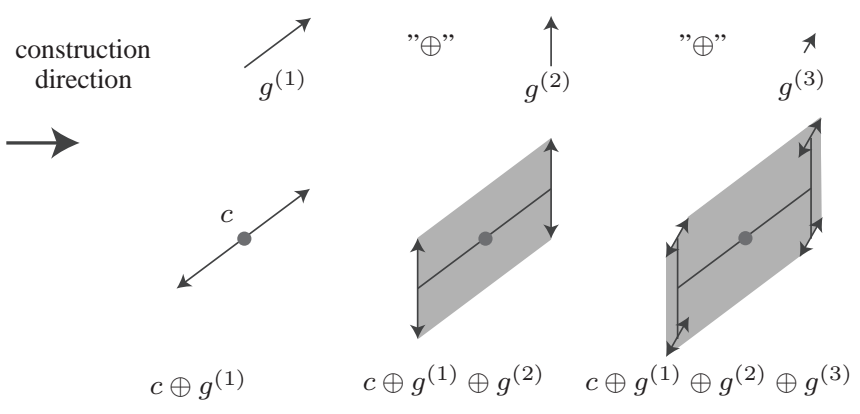

Fig. 2. Step-by-step construction of a two-dimensional zonotope.

The multiplication with a matrix $M \in \mathbb{R}^{o \times n}$ and the Minkowski addition of two zonotopes $\mathcal{Z}_{1}=\left(c, g^{(1)}, \ldots\right.$, $\left.g^{\left(p_{1}\right)}\right)$ and $\mathcal{Z}_{2}=\left(d, h^{(1)}, \ldots, h^{\left(p_{2}\right)}\right)$, are a direct consequence of the zonotope definition (see [47]):

$$
\begin{aligned}
\mathcal{Z}_{1} \oplus \mathcal{Z}_{2} & =\left(c+d, g^{(1)}, \ldots, g^{\left(p_{1}\right)}, h^{(1)}, \ldots, h^{\left(p_{2}\right)}\right) \\
M \otimes \mathcal{Z}_{1} & =\left(M c, M g^{(1)}, \ldots, M g^{\left(p_{1}\right)}\right)
\end{aligned}
$$

Additionally, the convex hull of $\mathcal{Z}_{1}$ and $e^{A r} \mathcal{Z}_{1}$ is required (see [46]):

$$
\begin{aligned}
& \mathrm{CH}\left(\mathcal{Z}_{1}, e^{A r} \mathcal{Z}_{1}\right) \subseteq \\
& \frac{1}{2}\left(c_{1}+e^{A r} c_{1}, g^{(1)}+e^{A r} g^{(1)}, \ldots, g^{\left(p_{1}\right)}+e^{A r} g^{\left(p_{1}\right)},\right. \\
& \left.\quad c_{1}-e^{A r} c_{1}, g^{(1)}-e^{A r} g^{(1)}, \ldots, g^{\left(p_{1}\right)}-e^{A r} g^{\left(p_{1}\right)}\right) .
\end{aligned}
$$

After introducing the matrix of generators $G=$ $\left[g^{(1)}, \ldots, g^{(p)}\right]$ and the alternative notation of a zonotope by $\mathcal{Z}=(c, G)$, the Cartesian product of two zonotopes $\mathcal{Z}_{1}=(c$, $G)$ and $\mathcal{Z}_{2}=(d, H)$ is

$$
\mathcal{Z}_{1} \times \mathcal{Z}_{2}=\left(\left[\begin{array}{l}
c \\
d
\end{array}\right],\left[\begin{array}{cc}
G & \mathbf{0} \\
\mathbf{0} & H
\end{array}\right]\right)
$$

where $\mathbf{0}$ is a matrix of zeros of proper dimension. For the multiplication of an interval matrix $\mathcal{M}$ with a zonotope, the matrix $\mathcal{M}$ is split into a real-valued matrix $M \in \mathcal{R}^{n \times n}$ and an interval matrix with radius $S \in \mathcal{R}^{n \times n}$, such that $\mathcal{M}=$ 
$M \oplus[-S, S]$. After introducing $S_{j}$ as the $j^{\text {th }}$ row of $S$, the result is overapproximated as shown in [23, Theorem 3.3] by

$$
\begin{aligned}
\mathcal{M Z}_{1} & \subseteq\left(M \mathcal{Z}_{1} \oplus[-S, S] \mathcal{Z}_{1}\right) \\
& \subseteq\left(M c_{1}, M g^{(1)}, \ldots, M g^{\left(p_{1}\right)}, h^{(1)}, \ldots, h^{(n)}\right) \\
h_{j}^{(i)} & =\left\{\begin{array}{l}
S_{j}\left(|c|+\sum_{k=1}^{p_{1}}|g|^{(k)}\right), \text { for } i=j \\
0, \text { for } i \neq j
\end{array} .\right.
\end{aligned}
$$

The overapproximative result of a quadratic map $\mathcal{Z}_{Q} \supseteq$ $\left\{\varphi \mid \varphi_{i}=x^{T} Q^{(i)} x, x \in \mathcal{Z}_{1}\right\}$ for a discrete set of matrices $Q^{(i)} \in \mathbb{R}^{n \times n}, i=1 \ldots n$, is computed according to [48] as

$$
\mathcal{Z}_{Q}=\left(d, h^{(1)}, \ldots, h^{(\sigma)}\right)
$$

with $\sigma=\left(\begin{array}{c}p+2 \\ 2_{T}\end{array}\right)-1$ generators, the center $d_{i}=c^{T} Q^{(i)} c+$ $0.5 \sum_{s=1}^{p} g^{(s)^{T}} Q^{(i)} g^{(s)}$ and the generators

$$
\begin{aligned}
j & =1 \ldots p: & h_{i}^{(j)} & =c^{T} Q^{(i)} g^{(j)}+g^{(j)^{T}} Q^{(i)} c \\
j & =1 \ldots p: & h_{i}^{(p+j)} & =0.5 g^{(j)^{T}} Q^{(i)} g^{(j)} \\
l & =\sum_{j=1}^{p-1} \sum_{k=j+1}^{p} 1: & h_{i}^{(2 p+l)} & =g^{(j)^{T}} Q^{(i)} g^{(k)}+g^{(k)^{T}} Q^{(i)} g^{(j)}
\end{aligned}
$$

The complexity of constructing this overapproximation with respect to the dimension $n$ is $\mathcal{O}\left(n^{5}\right)$.

\section{CAse Studies}

The introduced methods are applied to the transient stability analysis of power systems and to the analysis of uncertainty in renewable energy production. Transient stability analysis requires considering the nonlinearities of the dynamics since the operating condition is strongly perturbed. The analysis of the effects of uncertain energy production is presented in [28][30], but without considering nonlinear effects in contrast to the work presented here.

\section{A. Power System Modeling}

The mathematical models used for the case studies are standard models. The generator dynamics is borrowed from [28] and the power grid models are the IEEE 14-bus and 30-bus benchmark systems [49]. The dynamic variables of the $i^{\text {th }}$ generator are the generator phase angle $\tilde{\delta}_{i}[\mathrm{rad}]$, the angular velocity $\omega_{i}[\mathrm{rad} / \mathrm{s}]$, and the torque $T_{m, i}$ [p.u.] (p.u.: per unit). The commanded power production $P_{c, i}$ [p.u.] is a system input. The generator phase angles $\delta_{i}=\tilde{\delta}_{i}-\Theta_{s}$ are chosen relative to the slack bus angle $\Theta_{s}$ which has a constant angular velocity $\omega_{s}$ (in the previous work [31], the phase of the first generator is chosen as the reference phase). The dynamic equations of the chosen generator model are according to [28]:

$$
\begin{aligned}
\dot{\delta}_{i} & =\omega_{i}-\omega_{s} \\
\dot{\omega}_{i} & =-\frac{D_{i}}{M_{i}}\left(\omega_{i}-\omega_{s}\right)+\frac{1}{M_{i}} T_{m, i}-\frac{1}{M_{i}} P_{g, i} \\
\dot{T}_{m, i} & =-\frac{1}{T_{S V, i} R_{D, i} \omega_{s}}\left(\omega_{i}-\omega_{s}\right)-\frac{1}{T_{S V, i}} T_{m, i}+\frac{1}{T_{S V, i}} P_{c, i} .
\end{aligned}
$$

Parameters of each generator are the rotational inertia $M_{i}$ $\left[\mathrm{MJ} / \mathrm{Hz}^{2}\right]$, the damping coefficient $D_{i}[\mathrm{~s} / \mathrm{rad}]$, the time constant of the governor $T_{S V, i}[\mathrm{~s}]$, and the proportional gain of the governor $\frac{1}{R_{D, i}}$ [-]. For simplicity, the same model is used for all generators and synchronous condensers, where the latter are generators that produce no active power. The parameter values are chosen identical to [28] for each generator and synchronous condensers and are listed in Tab. I.

The power flow equations are obtained using standard methods, see e.g. [50, p.174]. The algebraic variables of the $i^{\text {th }}$ bus are the absolute value of the bus voltage $V_{i}$ [p.u], the phase angle of the bus voltage $\tilde{\Theta}_{i}[\mathrm{rad}]$, the active power $P_{i}$ [p.u.], the reactive power $Q_{i}$ [p.u.], and the generator voltage $E_{i}$ [p.u.] if the bus is connected to a generator. The bus phase angles with respect to the slack bus are denoted by $\Theta_{i}=\tilde{\Theta}_{i}-\Theta_{s}$. The buses are connected via admittances $Y_{i j}=Y_{j i}$, where $i$ and $j$ are the indices of the connected buses. The admittance from the generator to the $i^{\text {th }}$ generator bus is $Y_{g, i}$, where $\left|Y_{g, i}\right|$ [p.u.], $\Psi_{g, i}=\angle Y_{g, i}$ [rad] are the absolute values and phase angles, respectively. The absolute value and the angle of the admittances are denoted by $\left|Y_{i j}\right|$ and $\Psi_{i j}=\angle Y_{i j}$, respectively. The active and reactive power of each bus results from the generator production $P_{g, i}, Q_{g, i}$ and a demand of that node $P_{d, i}, Q_{d, i}$. The parameters of the power grid are chosen according to the corresponding IEEE benchmark problem and can be found in [49].

The numbering of the power network buses is renumbered from the original IEEE benchmark problems, where $N_{g}$ is the number of generators and $N_{l}$ is the number of load buses. In this work, the first bus $(i=1)$ is connected to a generator and serves as the slack bus. Further, the power system has $N_{g}$ socalled generator buses, which are connected to the generators. Those buses (including the slack bus) produce active and reactive power according to the following equations (see [50]):

$$
\begin{aligned}
P_{g, i} & =E_{i} V_{i}\left|Y_{g, i}\right| \cos \left(\Psi_{g, i}+\delta_{i}-\Theta_{i}\right)-V_{i}^{2}\left|Y_{g, i}\right| \cos \left(\Psi_{g, i}\right), \\
Q_{g, i} & =-E_{i} V_{i}\left|Y_{g, i}\right| \sin \left(\Psi_{g, i}+\delta_{i}-\Theta_{i}\right)+V_{i}^{2}\left|Y_{g, i}\right| \sin \left(\Psi_{g, i}\right) .
\end{aligned}
$$

The remaining $N_{l}$ buses are referred to as load buses ( $i=$ $\left.N_{g}+1 \ldots N_{g}+N_{l}\right)$. The power flow equations as in [50, p.174] of each bus are

$$
\begin{aligned}
P_{i} & =P_{g, i}+P_{g, i}^{d}+P_{d, i} \\
& =\sum_{j=1}^{N_{g}+N_{l}} V_{i} V_{j}\left|Y_{i j}\right| \cos \left(\Psi_{i j}+\Theta_{j}-\Theta_{i}\right), \\
Q_{i} & =Q_{g, i}+Q_{g, i}^{d}+Q_{d, i} \\
& =-\sum_{j=1}^{N_{g}+N_{l}} V_{i} V_{j}\left|Y_{i j}\right| \sin \left(\Psi_{i j}+\Theta_{j}-\Theta_{i}\right),
\end{aligned}
$$

where $P_{g, i}$ and $Q_{g, i}$ are the active and reactive power produced by generators with the dynamics according to (18), while $P_{g, i}^{d}$ and $Q_{g, i}^{d}$ are directly injected active and reactive powers from renewable energy sources. The power drop-out of the $i^{\text {th }}$ power plant is modeled by setting the active and reactive power in (19) and (18) to zero $\left(P_{g, i}=0, Q_{g, i}=0\right)$. In order to write the power system in the standard form of time-invariant, semi-explicit, index-1 DAEs as presented in (1), the dynamic, algebraic, and input variables are renamed. The following assignments are for a specific subsystem, i.e., the number of 
generators buses $N_{g}$ and the number of load buses $N_{l}$ are specific to the considered subsystem. Additionally, the number of cut transmission lines $N_{i}$ for the considered subsystem is introduced. It is also required to consider variables of neighboring subsystems. The $j^{\text {th }}$ voltage of the $k^{\text {th }}$ subsystem is denoted by $\hat{V}_{k, j}$ and an analogous notation is used for $\hat{\Theta}_{k, j}$. The function $[k, j]=h(i)$ returns the subsystem number $k$ of which the bus with number $j$ is connected to the considered subsystem and $i$ takes integers up to the number of cut transmission lines $\left(i=1 \ldots N_{i}\right)$, thus providing the first, second, and further input sources. The algebraic variables are assigned as follows:

$$
\begin{array}{ll}
i=1 \ldots N_{g}: & y_{i}=E_{i}, \\
i=1 \ldots N_{l}: & y_{N_{g}+i}=V_{N_{g}+i}, \\
i=2 \ldots\left(N_{g}+N_{l}\right): & y_{N_{g}+N_{l}+i-1}=\Theta_{i} .
\end{array}
$$

Note that $\Theta_{1}$ is not considered in the above assignment since it is the phase of the slack bus and thus always 0 . The dynamic variables are

$$
\begin{array}{ll}
i=1 \ldots N_{g}: & x_{i}=\delta_{i}, \\
i=1 \ldots N_{g}: & x_{N_{g}+i}=\omega_{i}, \\
i=1 \ldots N_{g}: & x_{2 N_{g}+i}=T_{m, i},
\end{array}
$$

and the inputs are assigned as follows:

$$
\begin{array}{ll}
i=1 \ldots N_{g}: & u_{i}=P_{c, i}, \\
i=1 \ldots\left(N_{g}+N_{l}\right): & u_{N_{g}+i}=P_{g, i}^{d}, \\
i=1 \ldots\left(N_{g}+N_{l}\right): & u_{2 N_{g}+N_{l}+i}=Q_{g, i}^{d}, \\
i=1 \ldots N_{i},[k, j]=h(i): & u_{3 N_{g}+2 N_{l}+i}=V_{k, j}, \\
i=1 \ldots N_{i},[k, j]=h(i): & u_{3 N_{g}+2 N_{l}+N_{i}+i}=\hat{\Theta}_{k, j} .
\end{array}
$$

When the $i^{\text {th }}$ power plant is not on the grid, the variable $E_{i}$ is removed from (19), (18), and is no longer an unknown variable. We replace $y_{i}=E_{i}$ by $y_{i}=V_{i}$ during the power drop-out, since the power plant can no longer control the voltage at the $i^{\text {th }}$ bus. All equations are automatically generated by symbolic computations in MATLAB to exclude errors during manual implementation.

TABLE I

PARAMETERS OF THE GENERATORS.

\begin{tabular}{cccccccc}
\hline$\forall i:$ & $M_{i}$ & $D_{i}$ & $\left|Y_{g, i}\right|$ & $\Psi_{g, i}$ & $T_{S V, i}$ & $R_{D, i}$ & $\omega_{s}$ \\
& $\frac{1}{15 \pi}$ & 0.04 & 5 & $-\frac{\pi}{2}$ & 1 & 0.05 & $120 \pi$ \\
\hline
\end{tabular}

\section{B. Transient Stability Analysis}

The transient stability analysis is performed as follows. After a pre-fault phase of $0.1 \mathrm{~s}$, the power plant producing the most power is taken off the grid (e.g. caused by a short circuit) for $0.03 \mathrm{~s}$ and afterwards reconnected. In the post-fault phase, the dynamics is computed until all continuous state variables reach the set of initial states. In all case studies, the center of the initial set is the steady state solution denoted by a superscripted zero. For all power generators the initial phase is $\delta_{i}(0) \in \delta_{i}^{0} \oplus 0.005 \cdot[-1,1]$, the initial rotational speed is $\omega_{i}(0) \in \omega_{i}^{0} \oplus 0.1 \cdot[-1,1]$, and the initial torque is $T_{m, i}(0) \in T_{m, i}^{0} \oplus 0.001 \cdot[-1,1]$.

The first case study is based on the IEEE 14-bus benchmark system, enhanced by the generator dynamics as introduced in
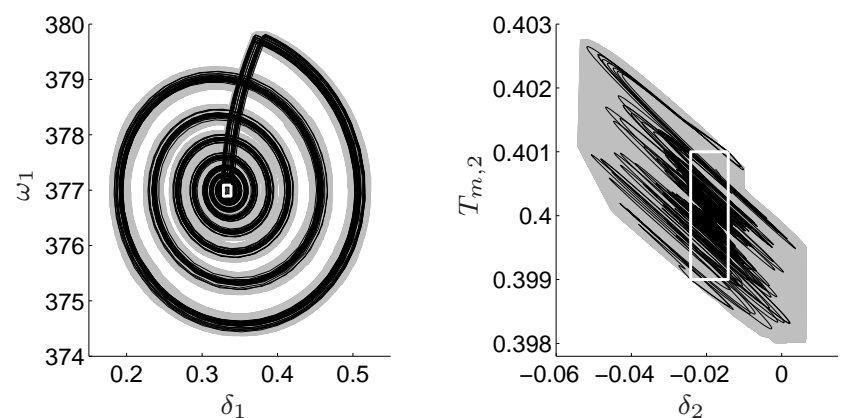

(a) Projections on differential variables of the 14-bus system.
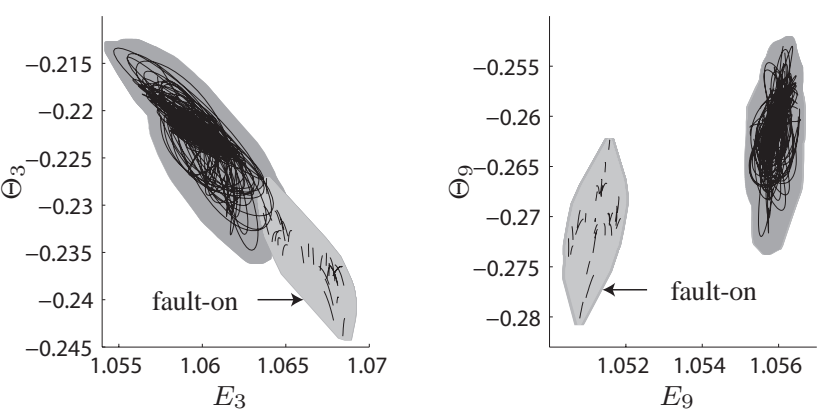

(b) Projections on algebraic variables of the 14-bus system.
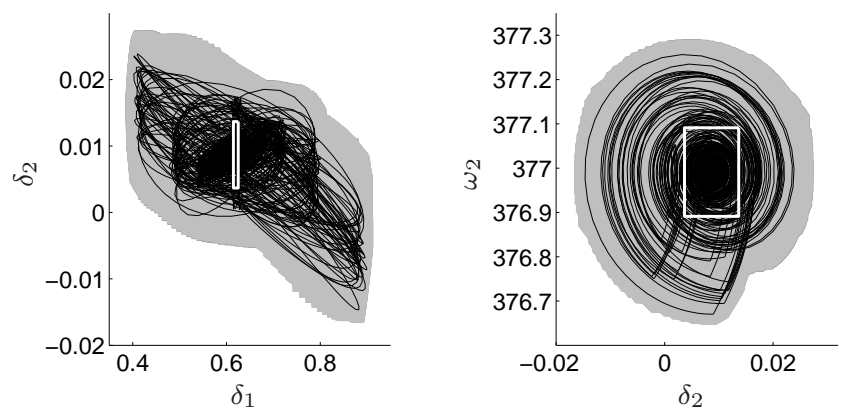

(c) Projections on differential variables of the 30-bus system.
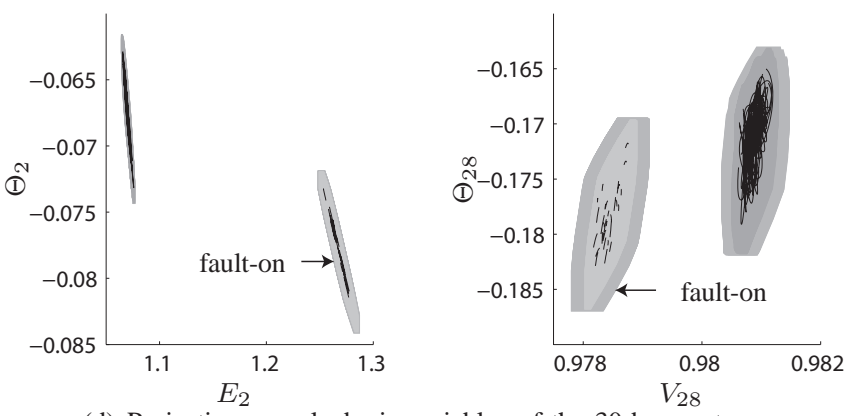

(d) Projections on algebraic variables of the 30-bus system.

Fig. 3. Selected projections of reachable sets for transient stability analysis. Black lines show random simulations, gray areas show reachable sets, and the white box the initial set. For algebraic variables, dark gray represents prefault and post-fault sets, light gray represents fault-on sets, and medium gray represents sets for all fault phases obtained by compositionally computing the linearization error.

Sec. VII-A. In that case study, it is investigated whether it is better to compute the reachable set of subsystems as described in Sec. V-A, or to compute the linearization error compositionally as described in Sec. V-B. For that purpose, the power system is split into two subsystems as shown in Fig. 5. The 
separate computation of the subsystems results in an explosion of the reachable set after $0.27 \mathrm{~s}$ in the considered case study (not illustrated due to space limitations). This is because the transmission line voltages and phase angles between interfaces of subsystems can be chosen arbitrarily within the uncertain sets $\hat{\mathcal{U}}^{(i)}$. This includes trajectories that excite oscillations, whereas all variables are correlated for the complete system, such that those behaviors are excluded in reality. However, when computing the linearization error compositionally, correlations between states are preserved. This is demonstrated by comparing the results of the monolithic and compositional linearization error computation for selected projections of the reachable set in Fig. 3. Although a different shade of gray is used to plot the reachable sets of the compositional approach for the linearization error computation, the difference can only be observed after zooming in for most projections. The accuracy of the results in Fig. 3 is indicated by simulations of system trajectories from randomly chosen initial states, which are plotted as black lines. Note that the results for the algebraic variables jump after the pre-fault and fault-on phase since the system model switches. Besides the 14-bus system, the compositional linearization error computation is also studied for the IEEE 30-bus benchmark system. Using the same generator models and parameters as for the 14-bus system, the reachable sets for the 30-bus system are presented in Fig. 3 for the full system and the partition into four subsystems (a figure showing the subsystems is not shown due to space limitations). Again, the overapproximation is marginal for most variables.

Although the overapproximation of the compositional computation of the linearization error is small, the savings in computation time are significant. This is because the linearization error computation consumes around $90 \%$ of the overall computation time. Thus, the compositional linearization error computation is clearly preferred over the compositional computation of the reachable set, since the latter results in significant overapproximation, while the savings in computational time are comparable for both methods. The computational times until all states return to the initial set (return time) using the compositional linearization error computation are listed in Tab. II for the considered case studies when the linearization errors of subsystems are computed in parallel using 4 cores. All computations are performed in MATLAB on an Intel XEON X5690 processor with $3.47 \mathrm{Ghz}$. Note that the 30-bus system can be computed in less time than the 14-bus system when using compositional linearization error computation with four subsystems. Considering that a single simulation takes around $1.5 \mathrm{~s}$ using the ode $15 \mathrm{~s}$ solver in MATLAB, the simulations of all corner cases of the 30-bus system with 18 dynamic variables requires $2^{18}$ simulations, which requires a computation time of $393216 \mathrm{~s}$, which takes around 100 times longer than the formal analysis.

\section{Critical Clearing Time}

Reachability analysis can also be used to determine the critical clearing time for a set of initial states rather than a single initial state. Since a single simulation run is faster than a complete reachability analysis, one should start determining the critical clearing time by simulating the system for different fault clearing times starting from the center of initial states $x^{0, c}$. The critical clearing time for $x^{0, c}$ is $t_{\text {crit }}=0.323 \mathrm{~s}$ for the previously considered IEEE 14-bus scenario in Sec. VII-B. When computing several simulations starting from the initial set specified in Sec. VII-B using the critical clearing time $t_{\text {crit }}=0.323 \mathrm{~s}$ obtained for $x^{0, c}$, some trajectories are stable and return to the original steady state, while other trajectories converge to another steady state as depicted in Fig. 4 for selected projections. The steady state from which the trajectories start is referred to as steady state $\alpha$, where steady state $\beta$ is the other possible steady state. The reachable set computation for this scenario computes until $t_{\text {crit }}=0.323 \mathrm{~s}$. Afterwards, the reachable set computation does not converge anymore and grows over all bounds. The reason is that trajectories diverge at the critical clearing time causing a large sensitivity for the algebraic state with respect to the dynamic state. As a consequence, the linearization error computations become very large so that the overapproximative computation of the linearization error no longer converges. In Fig. 4, the reachable set until the clearing time is shown for selected projections.

In order to determine the critical clearing time for all initial states, the recommended strategy is to start from the critical clearing time obtained by a single simulation and then iteratively decrease the critical clearing time until all states return to the original operating region as presented in Sec. VII-B.
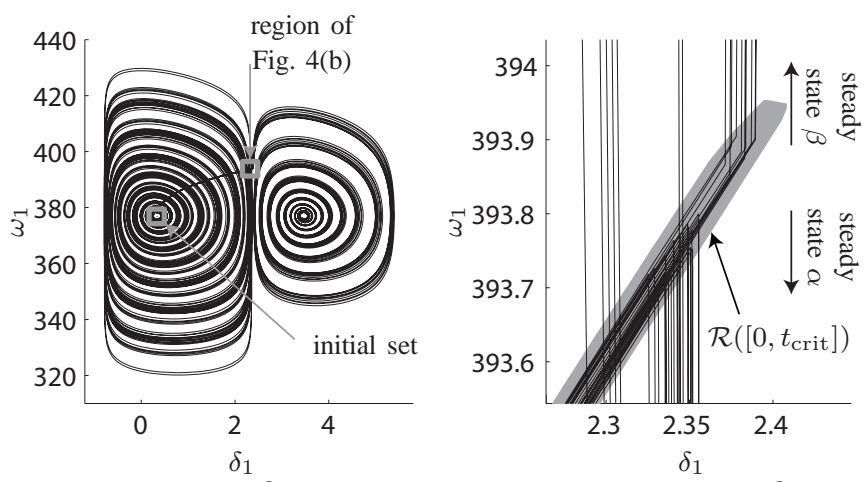

(a) Projection on $\delta_{1}, \omega_{1}$.

(b) Zoom of the projection on $\delta_{1}, \omega_{1}$.
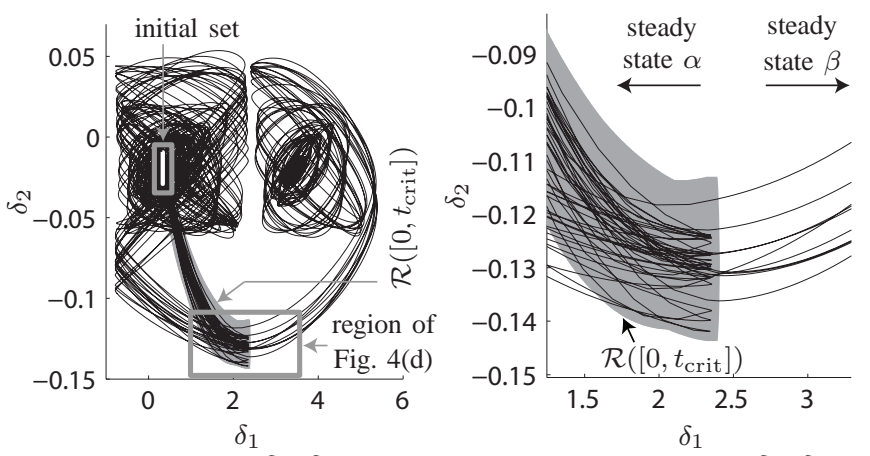

(c) Projection on $\delta_{1}, \delta_{2}$.

(d) Zoom of projection on $\delta_{1}, \delta_{2}$.

Fig. 4. Selected projections of reachable sets for the critical clearing time analysis of the IEEE 14-bus example. Black lines show random simulations for the time interval $t \in[0,4] \mathrm{s}$, gray areas show reachable sets until the critical clearing time ( $\left.t \in\left[0, t_{\text {crit }}\right]\right)$, and the white box the initial set. The right figures show zoomed regions within the left figures. 


\section{Effects of Variable Energy Production}

The effect of variable renewable energy production are demonstrated for the 14-bus system, where the generated active power at bus 13 and 14 are directly injected (see [28]), where $\forall t \in[0,5] s: P_{g, 13}^{d}(t), P_{g, 14}^{d}(t) \in\left\{\frac{t}{5} P^{*} \mid P^{*} \in\right.$ $[0.04,0.06]$ p.u. $\}$ modeling that the production uncertainty grows linearly over time. The conventional power plants produce only active power at bus 1 and 2: $P_{c, 1}=2$ [p.u.] and $P_{c, 2}=0.4$ [p.u.]. Selections on reachable sets over time for the time interval $[0,5] \mathrm{s}$ are presented in Fig. 6 together with random simulations for which a constant input is changed every $0.2 \mathrm{~s}$, causing jumps of algebraic variables.

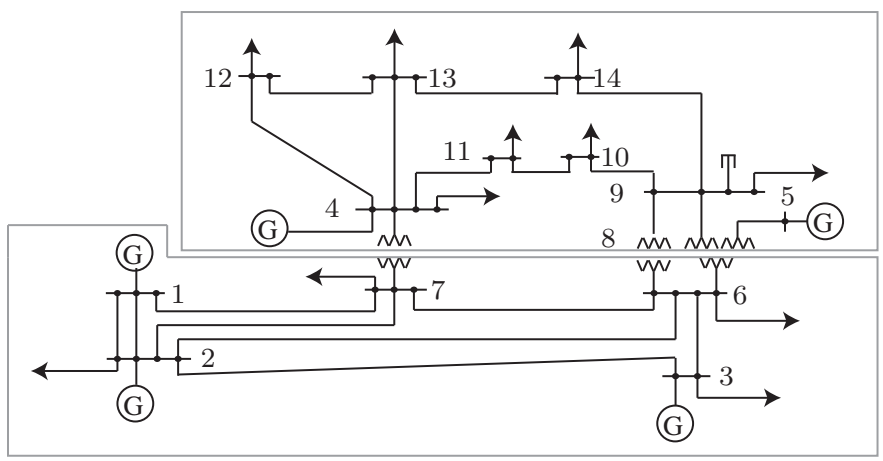

Fig. 5. IEEE 14-bus benchmark system. Gray lines show subsystem borders.

TABLE II

COMPUTATION TIMES AND TIMES TO RETURN TO INITIAL SET.

\begin{tabular}{lccccc}
\hline & IEEE & 14-bus & \multicolumn{3}{c}{ IEEE 30-bus } \\
subsystems & 1 & 2 & 1 & 2 & 4 \\
computation times & $1536 \mathrm{~s}$ & $853 \mathrm{~s}$ & $5002 \mathrm{~s}$ & $2025 \mathrm{~s}$ & $1428 \mathrm{~s}$ \\
return times & $4.0 \mathrm{~s}$ & $4.0 \mathrm{~s}$ & $4.4 \mathrm{~s}$ & $4.4 \mathrm{~s}$ & $4.4 \mathrm{~s}$ \\
\hline
\end{tabular}
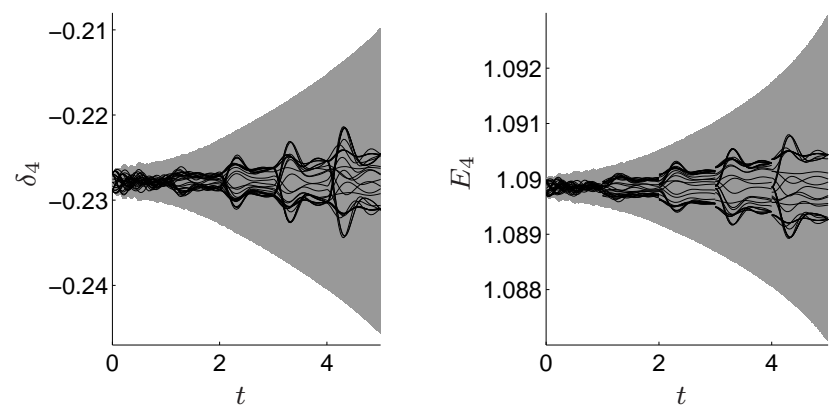

Fig. 6. Selected variable bounds over time due to variations in renewable energy production. Black lines show random simulations, the gray area shows the reachable sets.

\section{CONCLUSIONS}

The paper demonstrates a novel method to rigorously analyze power systems subject to varying operating conditions using reachability analysis. The consideration of semi-explicit, nonlinear, index-1 DAEs instead of ODEs makes it possible to not only analyze the frequency, but also the phases and voltages of each bus, which is especially useful to verify if cascading effects are avoided. The presented approach is based on an abstraction to linear differential inclusions, which is also of interest to other analysis techniques in power systems or for checking if linear models are justified. In order to improve the scalability, compositional techniques are proposed on the system level and on the level of the linearization error computation. The case studies revealed that the compositional computation of the linearization error is preferable since the computational savings are comparable, while the correlations between state variables are much better preserved.

The presented approach can be used for the same types of analysis for which simulation techniques are applied. Although a single simulation is computed faster than a reachable set, the simulation of all corner cases takes more time than the computation of the reachable set. For the 30-bus system, reachability analysis is around 100 times faster than simulating all corner cases due to the polynomial complexity of the presented approach. Note that the intention of the presented approach is not to replace simulation techniques, but complement them for a more rigorous analysis. Simulation techniques are especially useful to obtain a first idea about the system behavior, while reachability analysis can provide rigorous results in the presence of uncertainties.

A further application of reachability analysis is to compute the largest invariant set around a steady state [51], i.e., the largest set that cannot be left when starting in that set. Incorporating the proposed approach into an algorithm for computing the largest invariant set is part of future work.

\section{REFERENCES}

[1] P. Kundur, Power System Stability and Control. McGraw-Hill, 1994.

[2] K. Morison, L. Wang, and H. Hamadani, "New tools for blackout prevention," in Proc. of Power Systems Conference and Exposition, 2006, pp. $319-324$.

[3] M. L. Crow and M. Ilić, "The parallel implementation of the waveform relaxation method for transient stability simulations," IEEE Transactions on Power Systems, vol. 5, no. 3, pp. 922-932, 1990.

[4] J. Shu, W. Xue, and W. Zheng, "A parallel transient stability simulation for power systems," IEEE Transactions on Power Systems, vol. 20, no. 4, pp. 1709-1717, 2005

[5] V. Jalili-Marandi, Z. Zhou, and V. Dinavahi, "Large-scale transient stability simulation of electrical power systems on parallel GPUs," IEEE Transactions on Parallel and Distributed Systems, vol. 23, no. 7, pp. 1255-1266, 2012.

[6] M. La Scala, R. Sbrizzai, F. Torelli, and P. Scarpellini, "A tracking time domain simulator for real-time transient stability analysis," IEEE Transactions on Power Systems, vol. 13, no. 3, pp. 992-998, 1998.

[7] X. Yu and C. Singh, "A practical approach for integrated power system vulnerability analysis with protection failures," IEEE Transactions on Power Systems, vol. 19, no. 4, pp. 1811-1820, 2004.

[8] W. Wangdee and R. Billinton, "Bulk electric system well-being analysis using sequential Monte Carlo simulation," IEEE Transactions on Power Systems, vol. 21, no. 1, pp. 188-193, 2006.

[9] P. M. Anderson and A. Bose, "A probabilistic approach to power system stability analysis," IEEE Transactions on Power Apparatus and Systems, vol. 102, no. 8, pp. 2430-2439, 1983.

[10] M. Ribbens-Pavella and F. J. Evans, "Direct methods for studying dynamics of large-scale electric power systems - a survey," Automatica, vol. 21, no. 1, pp. 1-21, 1985.

[11] H.-D. Chiang, C.-C. Chu, and G. Cauley, "Direct stability analysis of electric power systems using energy functions: Theory, applications, and perspective," Proceedings of the IEEE, vol. 83, no. 11, pp. 1497-1529, 1995.

[12] M. Moechtar, T. C. Cheng, and L. Hu, "Transient stability of power system - a survey," in Proc. of the WESCON conference, 1995, pp. 166-171. 
[13] L. F. C. Alberto, F. H. J. R. Silva, and N. G. Bretas, "Direct methods for transient stability analysis in power systems: State of art and future perspectives," in Proc. of the IEEE Porto Power Tech Conference, 2001.

[14] U. Gabrijel and R. Mihalic, "Direct methods for transient stability assessment in power systems comprising controllable series devices," IEEE Transactions on Power Systems, vol. 17, no. 4, pp. 1116-1122, 2002.

[15] L. F. C. Alberto and H.-D. Chiang, "An uniform approach for direct transient stability analysis of electric power systems," in Proc. of the IEEE Power \& Energy Society General Meeting, 2009, pp. 1-7.

[16] A. R. Bergen, D. J. Hill, and C. L. de Marcot, "Lyapunov function for multimachine power systems with generator flux decay and voltage dependent loads," International Journal of Electrical Power \& Energy Systems, vol. 8, no. 1, pp. 2-10, 1986.

[17] P. W. Sauer, A. K. Behera, M. A. Pai, J. R. Winkelman, and J. H. Chow, "Trajectory approximation for direct energy methods that use sustained faults with detailed power system models," IEEE Transactions on Power Systems, vol. 4, no. 2, pp. 499-506, 1989

[18] I. A. Hiskens and D. J. Hill, "Energy functions, transient stability and voltage behavior in power systems with nonlinear loads," IEEE Transactions on Power Systems, vol. 4, no. 4, pp. 1525-1533, 1989.

[19] Y. Zou, M.-H. Yin, and H.-D. Chiang, "Theoretical foundation of the controlling UEP method for direct transient-stability analysis of network-preserving power system models," IEEE Transactions on Circuits and Systems I: Fundamental Theory and Applications, vol. 50, no. 10, pp. 1324-1336, 2003.

[20] A. Platzer and E. M. Clarke, "The image computation problem in hybrid systems model checking," in Hybrid Systems: Computation and Control, ser. LNCS 4416. Springer, 2007, p. 473486.

[21] E. Asarin, T. Dang, G. Frehse, A. Girard, C. Le Guernic, and O. Maler, "Recent progress in continuous and hybrid reachability analysis," in Proc. of the 2006 IEEE Conference on Computer Aided Control Systems Design, 2006, pp. 1582-1587.

[22] C. Le Guernic, "Reachability analysis of hybrid systems with linear continuous dynamics," Ph.D. dissertation, Univerité Joseph Fourier, 2009.

[23] M. Althoff, "Reachability analysis and its application to the safety assessment of autonomous cars," Dissertation, Technische Universität München, 2010, http://nbnresolving.de/urn/resolver.pl?urn:nbn:de:bvb:91-diss-20100715-963752$1-4$.

[24] S. Kaynama, "Scalable techniques for the computation of viable and reachable sets," Ph.D. dissertation, The University of British Columbia, 2012.

[25] L. Jin, H. Liu, R. Kumar, J. D. McCalley, N. Elia, and V. Ajjarapu, "Power system transient stability design using reachability based stability-region computation," in Proc. of the 37th Annual North American Power Symposium, 2005, pp. 338-343.

[26] L. Jin, R. Kumar, and N. Elia, "Reachability analysis based transient stability design in power systems," Electrical Power and Energy Systems, vol. 32, pp. 782-787, 2010

[27] Y. Susuki, T. Sakiyama, T. Ochi, T. Uemura, and T. Hikihara, "Verifying fault release control of power system via hybrid system reachability," in Proc. of the 40th North American Power Symposium, 2008.

[28] Y. C. Chen and A. D. Domínguez-García, "Assessing the impact of wind variability on power system small-signal reachability," in Proc. of the International Conference on System Sciences, 2011, pp. 1-8.

[29] _ - "A method to study the effect of renewable resource variability on power system dynamics," IEEE Transactions on Power Systems, vol. 27, no. 4, pp. 1978-1989, 2012.

[30] H. N. Villegas Pico, D. C. Aliprantis, and E. C. Hoff, "Reachability analysis of power system frequency dynamics with new high-capacity HVAC and HVDC transmission lines," in Proc. of the IREP Bulk Power System Dynamics and Control Symposium, 2013.

[31] M. Althoff, M. Cvetković, and M. Ilić, "Transient stability analysis by reachable set computation," in Proc. of the IEEE PES Conference on Innovative Smart Grid Technologies Europe, 2012.

[32] J. Machowski, J. Bialek, and J. Bumby, Power System Dynamics: Stability and Control. Wiley, 2008.

[33] M. Althoff, O. Stursberg, and M. Buss, "Reachability analysis of nonlinear systems with uncertain parameters using conservative linearization," in Proc. of the 47th IEEE Conference on Decision and Control, 2008, pp. 4042-4048.

[34] K. Makino and M. Berz, "Rigorous integration of flows and ODEs using Taylor models," in Proc. of Symbolic-Numeric Computation, 2009, pp. 79-84.
[35] G. Frehse, C. L. Guernic, A. Donzé, S. Cotton, R. Ray, O. Lebeltel, R. Ripado, A. Girard, T. Dang, and O. Maler, "SpaceEx: Scalable verification of hybrid systems," in Proc. of the 23rd International Conference on Computer Aided Verification, ser. LNCS 6806. Springer, 2011, pp. 379-395.

[36] M. Althoff, C. Le Guernic, and B. H. Krogh, "Reachable set computation for uncertain time-varying linear systems," in Hybrid Systems: Computation and Control, 2011, pp. 93-102.

[37] M. Berz and G. Hoffstätter, "Computation and application of Taylor polynomials with interval remainder bounds," Reliable Computing, vol. 4, pp. 83-97, 1998.

[38] S. B. Yusof, G. J. Rogers, and R. T. H. Alden, "Slow coherency based network partitioning including load buses," IEEE Transactions on Power Systems, vol. 8, no. 3, pp. 1375-1382, 1993.

[39] E. De Tuglie, S. M. Iannone, and F. Torelli, "A coherency recognition based on structural decomposition procedure," IEEE Transactions on Power Systems, vol. 23, no. 2, pp. 555-563, 2008.

[40] C. S. Chang, L. R. Lu, and F. S. Wen, "Power system network partitioning using tabu search," Electric Power Systems Research, vol. 49, no. 1, pp. 55-61, 1999.

[41] A. Chutinan and B. H. Krogh, "Computational techniques for hybrid system verification," IEEE Transactions on Automatic Control, vol. 48, no. 1 , pp. 64-75, 2003.

[42] A. Girard, C. Le Guernic, and O. Maler, "Efficient computation of reachable sets of linear time-invariant systems with inputs," in Hybrid Systems: Computation and Control, ser. LNCS 3927pl. Springer, 2006, pp. 257-271.

[43] A. B. Kurzhanski and P. Varaiya, "Ellipsoidal techniques for reachability analysis," in Hybrid Systems: Computation and Control, ser. LNCS 1790. Springer, 2000, pp. 202-214.

[44] A. Girard and C. Le Guernic, "Efficient reachability analysis for linear systems using support functions," in Proc. of the 17th IFAC World Congress, 2008, pp. 8966-8971.

[45] O. Stursberg and B. H. Krogh, "Efficient representation and computation of reachable sets for hybrid systems," in Hybrid Systems: Computation and Control, ser. LNCS 2623. Springer, 2003, pp. 482-497.

[46] A. Girard, "Reachability of uncertain linear systems using zonotopes," in Hybrid Systems: Computation and Control, ser. LNCS 3414. Springer, 2005, pp. 291-305.

[47] W. Kühn, "Rigorously computed orbits of dynamical systems without the wrapping effect," Computing, vol. 61, pp. 47-67, 1998.

[48] M. Althoff and B. H. Krogh, "Avoiding geometric intersection operations in reachability analysis of hybrid systems," in Hybrid Systems: Computation and Control, 2012, pp. 45-54.

[49] University of Washington, "Power systems test case archive," http: //www.ee.washington.edu/research/pstca/, University of Washington.

[50] P. Schavemaker and L. van der Sluis, Electrical Power System Essentials. Wiley, 2008.

[51] S. V. Raković, P. Grieder, M. Kvasnica, D. Q. Mayne, and M. Morari, "Computation of invariant sets for piecewise affine discrete time systems subject to bounded disturbances," in Proc. of the 43rd IEEE Conference on Decision and Control, 2004, pp. 1418-1423.

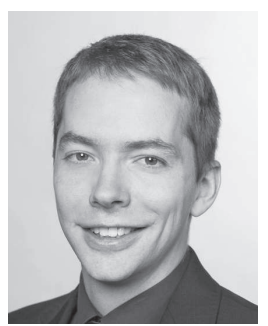

Matthias Althoff is assistant professor in computer science at Technische Universität München, Germany. He received the diploma engineering degree in Mechanical Engineering in 2005, and the Ph.D. degree in Electrical Engineering in 2010, both from Technische Universität München, Germany. From 2010 to 2012 he was a postdoctoral researcher at Carnegie Mellon University, Pittsburgh, USA, and from 2012 to 2013 an assistant professor at Technische Universität Ilmenau, Germany. His research interests include formal verification of continuous and hybrid systems, reachability analysis, planning algorithms, nonlinear control, automated vehicles, and power systems. 